\title{
DEFECTS IN LONG BONES FROM SEVERE NEGLECTED OSTEITIS
}

\author{
J. C. Griffiths, Salford, England
}

Osteitis has been transformed in recent times from a lethal or crippling disease to one in which restitution of bone to normal can be expected (Trueta and Morgan 1954). Yet if diagnosis and treatment are delayed the disease, despite antibiotics, may show all its former complications and may run the same unfavourable course as was described by earlier surgeons (Pyrah and Pain 1933). In Africa and other underdeveloped countries osteitis still presents in the classical manner (Bryson and Mandell 1964) and the problem of complete bone destruction, exceptionally rare elsewhere, is still encountered. Thus it is not surprising that of the twelve patients to be described with this complication eleven were seen in the course of one year spent in Kenya (1965-66).

\section{MATERIAL}

Relevant details of the twelve patients are summarised in Table I. The follow-up on most of the patients was, because of an African environment, unavoidably shorter than was desirable but all had gone through the initial stages satisfactorily and there is adequate evidence from other authors of late success in similar patients. In some patients, many of whom were referred for treatment from elsewhere, the early records were scanty and the radiographs missing. This is regrettable because precise details about the types of organism, antibiotic sensitivity, antibiotics used and duration of use, and the methods and efficiency of immobilisation would have been instructive.

\section{ILLUSTRATIVE CASE REPORTS}

Case 1: tibial defect-A boy of eight years was first seen in 1963 with a history of pain, swelling and discharge in the right leg for one month. Extensive radiological changes were seen in the tibia (Fig. 1); pathological fractures had occurred at both ends of a massive sequestrum and the involucrum was incomplete. There was already evidence of proximal fibular subluxation.

Operations and progress-The sequestrum was removed two months later, after a period of plaster immobilisation and antibiotic therapy; the wounds healed by first intention. Two years later there was still no evidence of regeneration and the bone ends had become smooth and pointed. The fibula was hypertrophied and dislocated superiorly. The epiphyses of the tibia still seemed open. Two-stage transfer of the fibula was therefore carried out, with screw fixation. There was a temporary drop foot after the proximal transfer. Six months later bony union had occurred and the drop foot had recovered (Fig. 2). Growth prospects were good radiologically although the limb at this stage was four centimetres short.

Case 2: tibial defect-A girl from the Hebrides, first seen at the age of sixteen months, is the only one in this series who was treated in the United Kingdom. Fulminating osteitis of the right tibia developed in 1958 and was treated with penicillin, but a week later there was little response and the child was transferred to Glasgow for further treatment. Three months later the whole tibial diaphysis was seen to be sequestrating and there was not an adequate involucrum.

Operations and progress-Sequestrectomy was performed twice and was followed by primary wound healing. No regeneration of the tibia occurred (Fig. 3) and after a year a two-stage fibular transfer was done, with a four-month interval between stages. The proximal transfer presented some technical difficulties: internal fixation was not used and the transfer slipped on the first occasion and had to be redone. A year later the transfer had fused but already there was evidence of closure of both tibial epiphyses. Varus deformity at the knee developed three years later and necessitated corrective osteotomy through the transplanted fibula. The fibula hypertrophied very well (Fig. 4) but the limb is now nine centimetres short and a Syme's amputation is contemplated. 


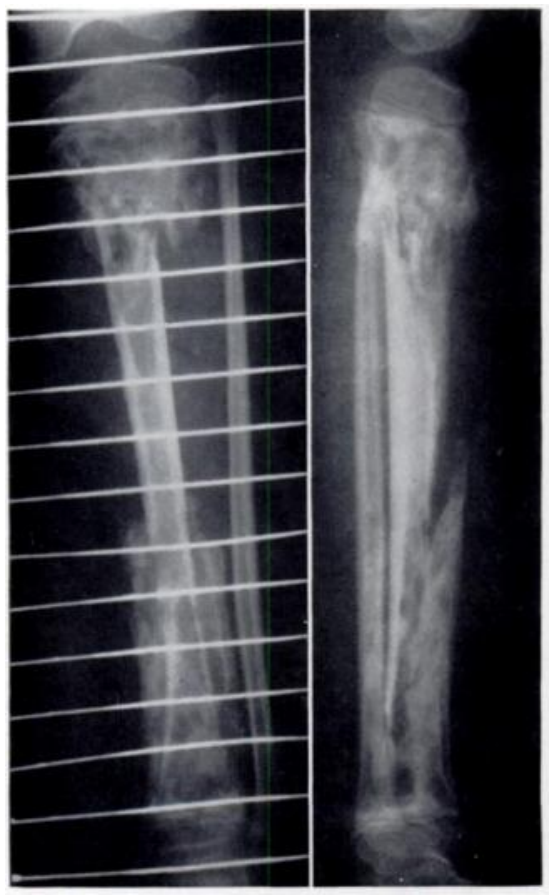

Fig. 1

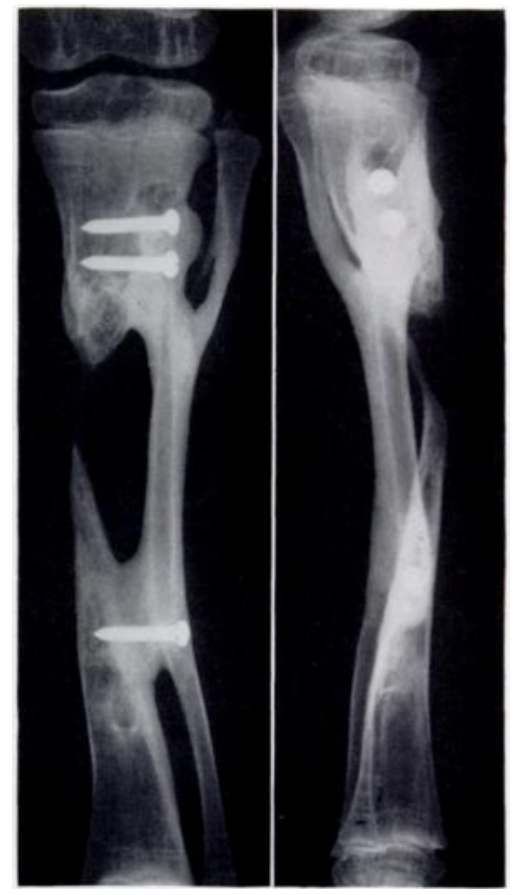

Fig. 2

Case 1. Figure 1-Radiographs before sequestrectomy. Figure 2-Six months after fibular transfer.

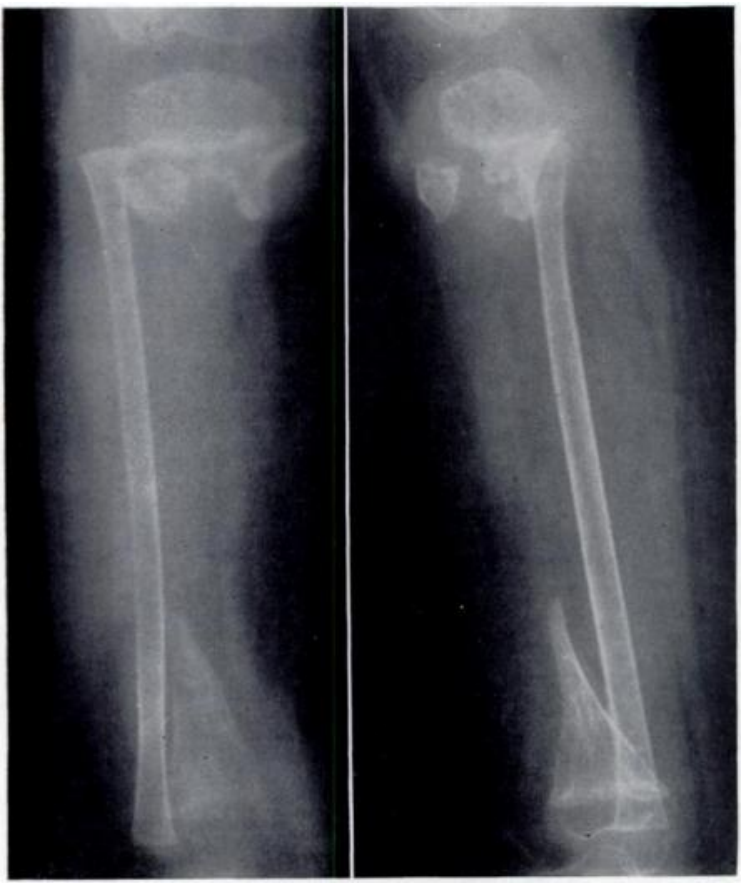

Fig. 3

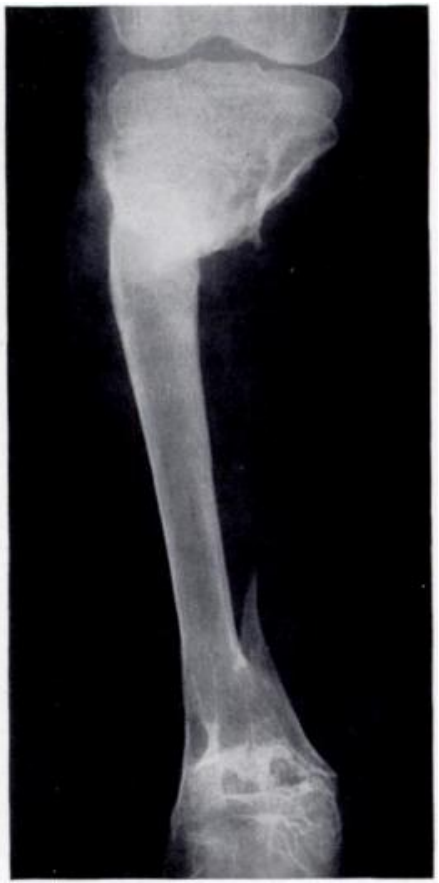

FIG. 4

Case 2. Figure 3-Shortly after sequestrectomy. Figure 4-Five years after fibular transfer; slight persistent genu varum is still apparent. 


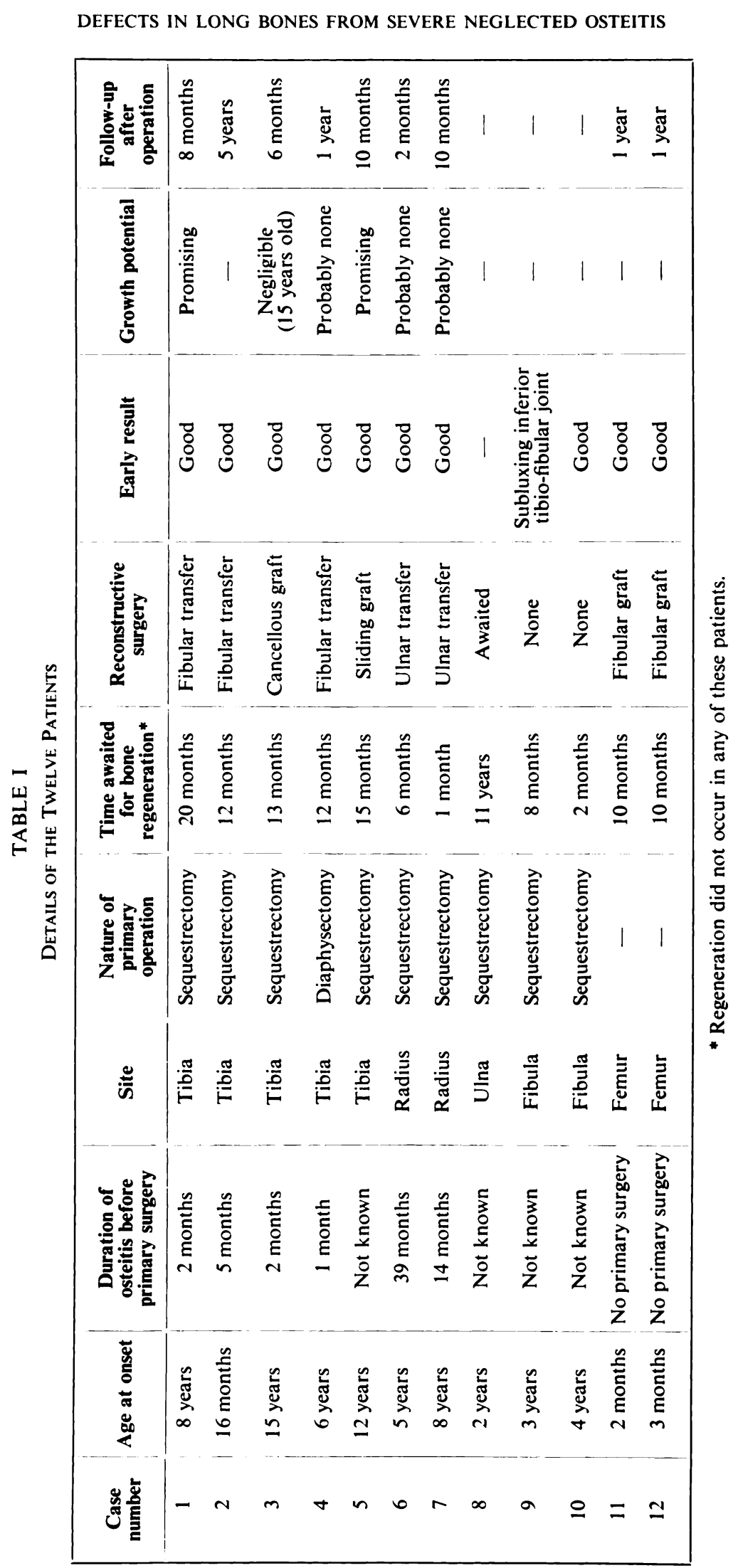

VOL. 50 B, NO. 4, NOVEMBER 1968 
Case 3: tibial defect-A boy aged fifteen years presented with acute osteitis in 1964. At three months and again at four months attempts at sequestrectomy were made, the final attempt resulting in a fracture.

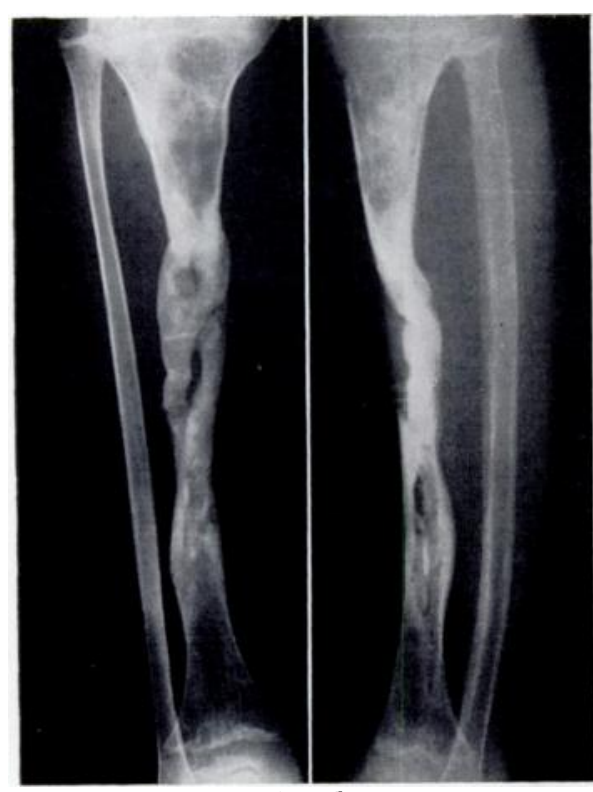

Fig. 5

Case 3-Five months after cancellous grafting.

Radiographs up to this point are missing. The patient was first seen a year and a quarter after the initial osteitis with an established gap of about seven centimetres, a hypertrophied fibula and proximal fibular dislocation.

Operations and progress-After a small sinus had healed, a bridging eight-hole bone plate was applied and the gap filled with bone chips from the ilium. A further sinus developed, but the urge to remove the plate was resisted until two months had passed. At this stage most of the grafts had incorporated. Five months after the operation the grafts were consolidating and the tibia was clinically solid (Fig. 5). A year later a further sinus appeared although bony consolidation was progressing. The proximal tibial epiphysis is now closed but shortening is slight and in view of the patient's age is not likely to increase. In a patient of this age with a relatively short gap a direct graft was permissible, but there would probably have been less trouble with sepsis if a fibular transfer had been done.

Case 6: defect of radius-A boy of five years gave a history of swelling and discharge in the left forearm for three years. Radiographs showed sequestration of almost the whole of the radial diaphysis and there was no evidence of an involucrum (Fig. 6). Three months of immobilisation and antibiotic therapy were ineffective.

Operations and progress-Sequestrectomy was performed and was followed by primary wound healing and disappearance of the sinus. Four months later distal radio-ulnar fusion was carried out without complication, and union occurred within two months (Fig. 7). The growth potential of the distal remnant of radius is very doubtful.

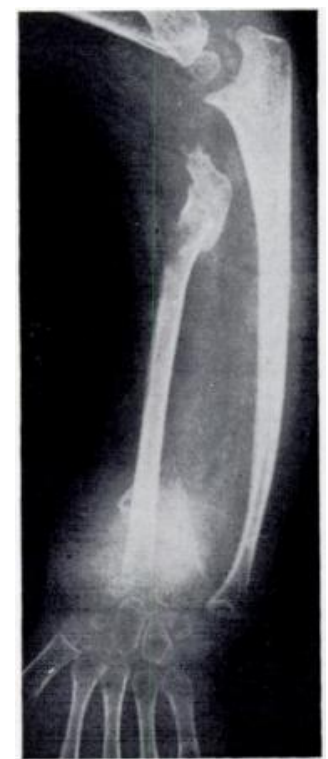

FIG. 6

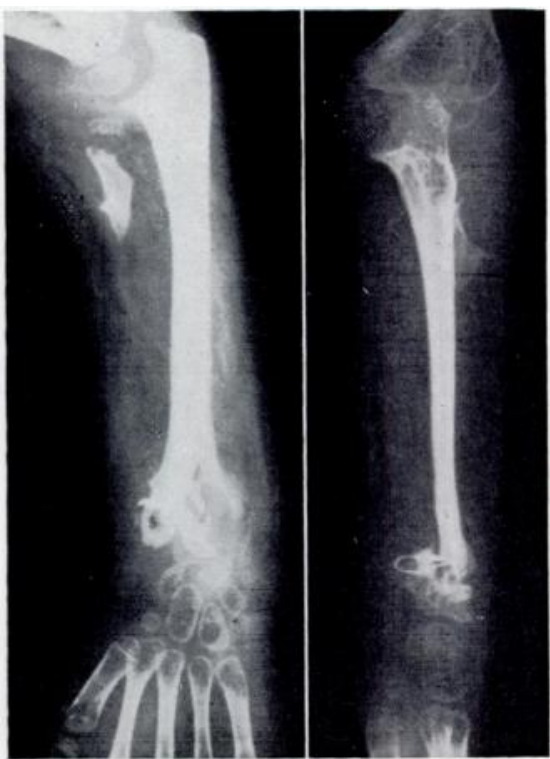

FIG. 7

Case 6. Figure 6-Before sequestrectomy. Figure 7-Two months after distal radio-ulnar fusion.

Case 8: defect of ulna-A girl was first seen at the age of thirteen with a history of an operation for osteitis of the ulna ten years before. There was much cosmetic deformity of the elbow and the 
radiographs showed a severely distorted radio-humeral joint (Fig. 8). She had excellent flexion and extension in the elbow but hardly any rotation. It is of interest to note the slight functional disability in spite of the partial absence of the ulna for so many years. It was thought that the disability was not bad enough to warrant operation.

Case 9: defect of fibula-A girl of three years had an acute osteitis of the fibula which presented with an abscess. This was drained, and penicillin and streptomycin were given for two weeks. A sinus developed and radiographs showed sequestration of the whole fibular diaphysis; there was no involucrum but there was early evidence of subluxation proximally of the lower end of the fibula.

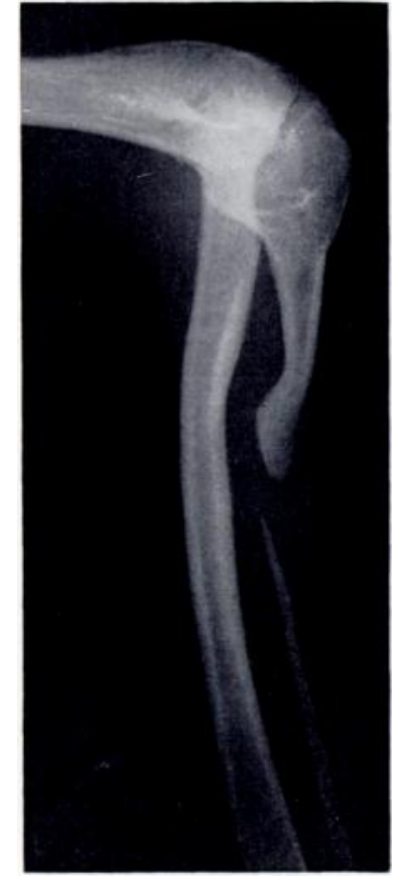

Fig. 8

Case 8-Defects after osteitis of the ulna.

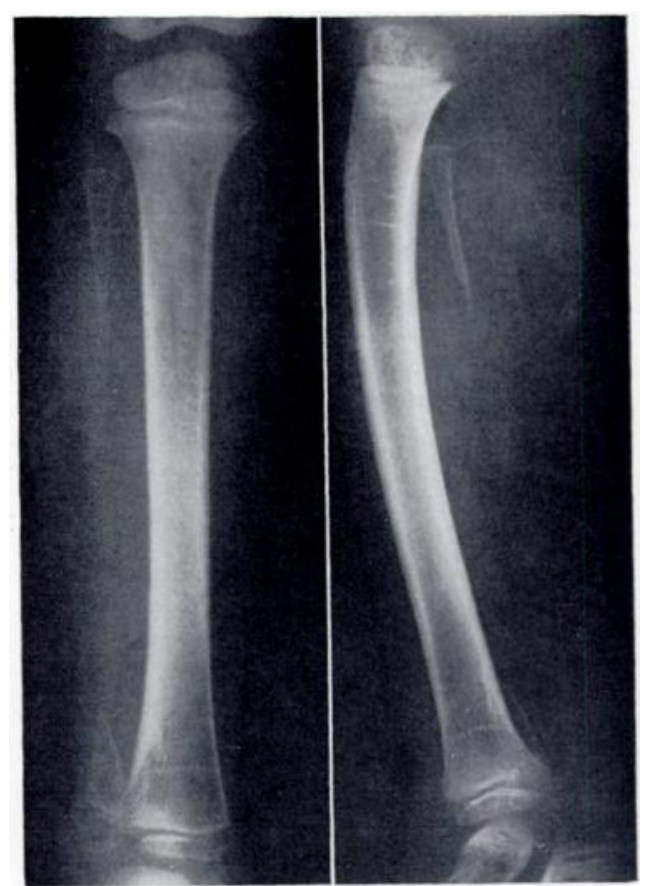

FIG. 9

Case 9-Eight months after sequestrectomy. There is inferior tibio-fibular subluxation.

Operations and progress-Sequestrectomy was followed by primary wound healing. Eight months later there was no evidence of fibular regeneration, and the inferior tibio-fibular joint was subluxated (Fig. 9). A slight valgus deformity has occurred at the ankle.

Case 11 : defect of femur-A girl had severe femoral osteitis at the age of two or three months. Antibiotic treatment was given for an unknown period; a sinus developed which closed at nine months; there were no operations. When first seen the infection had settled but there was a large gap in the femoral shaft and destruction of the head of femur (Fig. 10). The distal end of femur consisted of the epiphysis only, with no metaphysial zone.

Operation and progress-A piece of fibula from the normal leg was wedged into the gap and appeared to be incorporated one year later (Fig. 11). Clinically the femur is now firm, but the knee joint is abnormally mobile. Further longitudinal femoral growth is unlikely.

Case 12: defect of femur-A girl developed severe femoral osteitis at the age of three months which settled with antibiotics and simple traction three months later. A small sinus developed and then closed without surgery. When first seen there was an established gap in the femur, a normal femoral capital epiphysis and an isolated distal femoral epiphysis surmounted by a rudimentary metaphysial cap (Fig. 12).

Operations and progress-A fibular graft was inserted but it proved impossible to fix it firmly to the fragment of metaphysis except by insertion into the epiphysis, which made further growth doubtful. The graft has incorporated well and the knee is stable (Fig. 13).

VOL. $50 \mathrm{~B}$, NO. 4, NOVEMBER 1968 


\section{ETIOLOGICAL CLASSIFICATION}

The patients in this series fall clearly into three groups: primary diaphysectomy, late sequestrectomy and neonatal disease.

Primary diaphysectomy-Only one patient (Case 4) had been subjected to primary diaphysectomy. Before antibiotics this operation was favoured by some as a curative or life-saving measure but, in general, most opposed it (Platt 1928, White 1935) because of the

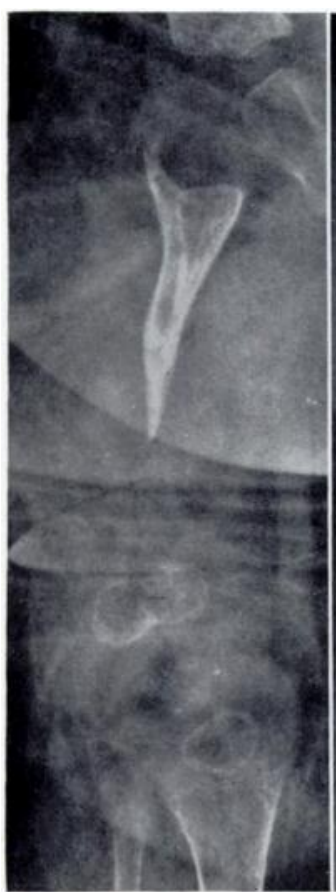

Fig. 10

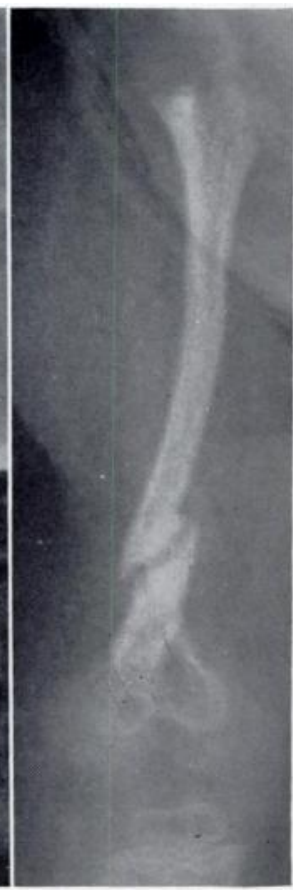

FIG. 11

Case 11. Figure 10-A femoral defect but the distal epiphysis is still present. Figure 11-One year after a fibular graft in which a fracture had occurred but is here shown healing. complex reconstructive problems which followed any subsequent failure of bone regeneration. A proportion of bones did reform, however, and to this end emphasis was placed on preserving and suturing the periosteum after diaphysectomy (Phemister 1915) and on the observation (Bosworth 1933) that regeneration was more likely to occur in patients under six years of age. Although isolated reports of successful diaphysectomy even without an attempt to preserve the periosteum have been made (Evans 1914) the procedure generally has unpredictable results and is certainly not justifiable when antibiotics are available. Osteitis of the fibula can be an exception to this rule (Bailey 1930) but even then care must be taken to preserve the integrity of the inferior tibio-fibular syndesmosis, because a valgus ankle may follow proximal migration of the distal fibular remnant (Butler 1940) (Case 9).

Late sequestrectomy-Most of the patients in this series failed to develop an involucrum. Consequently sequestrectomy had to be undertaken in the full knowledge that a large bone defect would result. This situation cannot be readily avoided because it implies basic deficiencies in early diagnosis and treatment with consequent thrombosis of two sets of vessels, nutrient and periosteal. One patient, however (Case 3), had radical treatment performed too early and the case illustrates the complications of over-enthusiastic surgery in the subacute phase of severe osteitis (Roper 1964). In the earlier stages of the disease emphasis should be placed on conservatism in the hope that an involucrum will develop. This implies not only adequate antibiotic therapy but also suitable immobilisation to prevent fracture, non-union and resorption of bone (Capener and Pierce 1932).

Neonatal disease-The literature reveals no previous record of bone defects from neonatal osteitis. The two such patients reported here presented unusual features. Firstly, no sequestrum was seen or removed, although both patients had healed sinus scars on the thigh through which pus and bone fragments may have been extruded. Secondly, there was no evidence of involucrum formation. This would be expected to be more than adequate at this age (Trueta 1959). No further comment can be made except to note that further support is given to suggestions that neonatal osteitis is not always benign (Thomson and Lewis 1950) and that destruction of the femoral head is a feature (Trueta 1959) which will aggravate the late prognosis (Case 11, Fig. 10).

\section{MANAGEMENT}

It was usual for the patient to present with an established defect or with a large chronic sequestrum without an involucrum. There is little to be gained by delaying reconstruction 


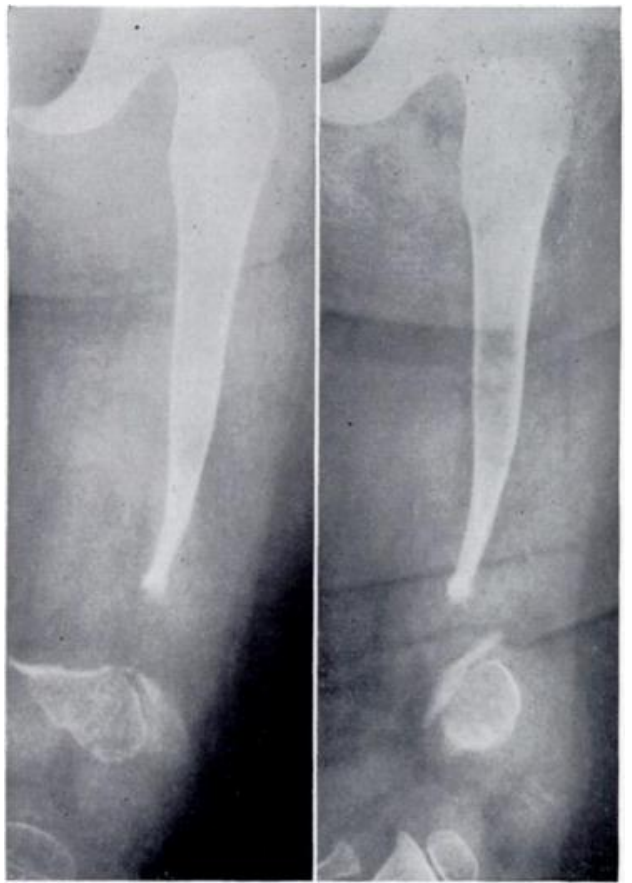

FIG. 12

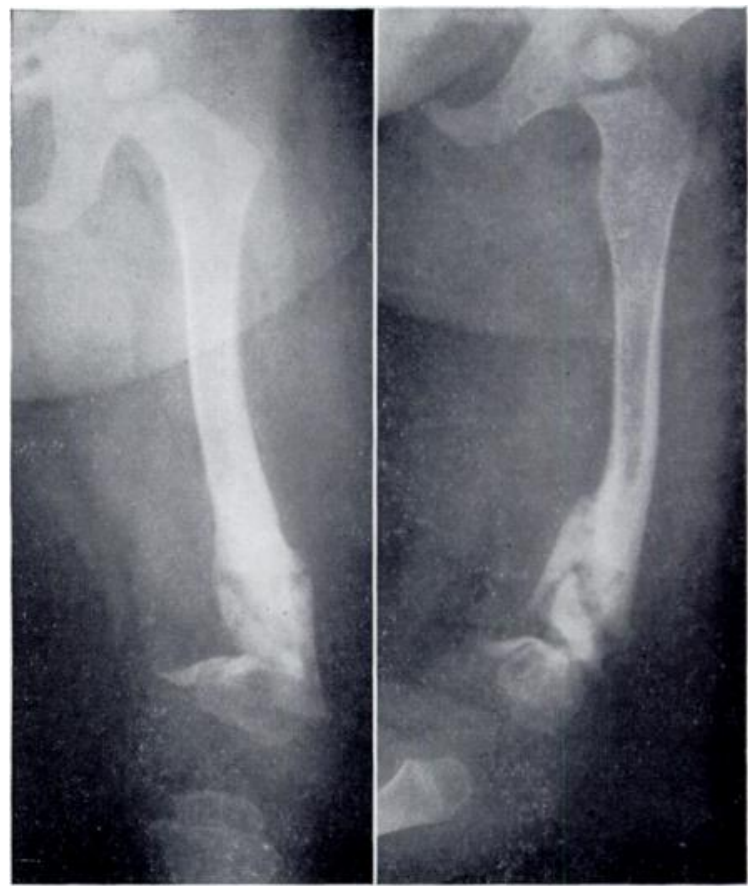

Fig. 13

Case 12. Figure 12-Femoral defect but the metaphysial remnant is clearly seen. Figure 13-One year after a fibular graft which fractured but is healing.

longer than necessary for healing of wounds and sinuses. If, after primary diaphysectomy, no signs of regeneration appear within six months (Bosworth 1933) the gap is likely to be permanent. If a sequestrum is present but with no involucrum and a long history conservative treatment is futile. Early sequestrectomy not only allows sinuses to heal and makes subsequent reconstruction possible but may also help to preserve the epiphysial growth zone from the adverse effects of continuing infection. Removal of a sequestrum will not be followed by spontaneous bone regeneration-although in Case 1 it was awaited for two years. Therefore reconstruction should be early because removal of the sequestrum, which can support the soft tissues, will increase deformity, especially in the forearm.

Reconstruction can be on two lines. There is no alternative to direct grafting except in the forearm or leg where transference of the unaffected bone is possible. Fortunately it is rare for both bones to be affected at once. Descriptions of this method appeared early (Hahn 1884) because it offered an indirect approach to an infected area before antibiotics were available. Successful transference of the fibula to the tibial remnants, usually in two stages and by varying techniques, has been described by Huntington (1905), Girdlestone and Foley (1933), Wilson (1941), Lucas (1960), Parisien (1963), Ruskowski (1965) and Bosworth, Liebler, Nastasi and Hamada (1966). Similarly, in the forearm synostosis between distal radius and proximal ulna to produce a forearm with a single bone and no rotation has often been described (Groves 1921, Greenwood 1932, Jones 1934, Vitale 1952, Murray 1955, Lowe 1963).

Direct grafting of a radial defect was reported by Gray (1928), but it was not until antibiotics became available that it was found possible to use small bone grafts reliably in the presence of established or potential sepsis (Mowlem 1944, Hazlett 1954). However, the potential risks are illustrated by Case 3 in which, although the gap was closed successfully, there was persistent sepsis in the early stages, followed at one year by recurrence of a sinus. When possible, bone transference is preferable to direct grafting unless the bone defect is small.

VOl. 50 B, NO. 4, NOVEMBer 1968 


\section{PROGNOSIS}

In the forearm the results of bone transference for either a radial or an ulnar defect are good. Direct grafting has nothing to offer since it does little to improve the cosmetic defect and is also less likely to succeed. Loss of pronation and supination is inevitable whatever method is used, because of dislocation either of the inferior or of the superior radio-ulnar joint (Jones 1934). Operation is needed particularly for radial defects: function can be good for many years with an ulnar defect although the appearance is poor.

There is ample evidence for the success of fibular transfer for tibial defects. Early complications were two cases of common peroneal nerve palsy which both recovered in a few weeks, and varus deformity at the knee from insertion of the upper end of the fibula too laterally in the proximal tibia. Failure of longitudinal growth is an important limiting factor and in some cases may make subsequent amputation advisable. Nevertheless there is a considerable difference between a Syme's amputation and one made through or above the knee. A simple "elephant" boot for a Syme's amputation is acceptable to the African and is much better and cheaper than a complex prosthesis required for a higher amputation. Preservation of an axial bone below the knee is the only means whereby such a conservative amputation can be made possible.

Longitudinal growth in the femora of the two neonates reported here is bound to fail. The attempt to preserve at least a few inches of stump preparatory to an early through-knee amputation is, however, thoroughly justifiable.

\section{SUMMARY}

1. Because of socio-medical deficiencies, osteitis in some parts of the world still conforms to the classical pattern seen before the introduction of antibiotics.

2. One of many complications is loss of continuity from widespread destruction of tubular bone.

3. Twelve patients with this complication are described. The most satisfactory treatment, when practicable, is the transference of an adjoining normal bone into the remnants of the defective one.

4. Two neonates each with a massive defect of the femur are described. This complication of osteitis at this age has not been reported before.

5. The long-term prognosis of lower limb defects is poor when there is failure of bone growth. Nevertheless, reconstructive procedures are still worth attempting because later amputation, if necessary, can be conservative, and prosthetic difficulties may thus be eased.

Grateful acknowledgments are accorded to Professor $\mathrm{R}$. Barnes and $\mathrm{Mr} \mathrm{J}$. T. Brown for generous advice and criticism. I should like to express my thanks to the staff of the Shirate Mission Hospital, the African Medical and Research Foundation and the Association for the Physically Disabled of Kenva for help in arranging the treatment and follow-up of two of the patients. Thanks are also due to $\mathrm{MrC}$. Da Cunha, surgeon at the Provincial Hospital, Nakuru, Kenya, for details of Case 5. I am indebted to Mr N. J. Blockey for recent information about Cases 3,11 and 12. The illustrations were prepared by Mr G. Donald and the staff of the Medical Illustrations Department, Western Infirmary, Glasgow.

\section{REFERENCES}

BaIIEY, H. (1930): Diaphysectomy and Primary Suture for Acute Osteomyelitis of the Fibula. British Journal of Surgery, 17, 641.

Bosworth, D. M. (1933): Subperiosteal Resection of the Tibial Shaft in Osteomyelitis. Journal of the American Medical Association, 101, 1542.

Bosworth, D. M., Liebler, W. A., Nastasi, A. A., and Hamada, K. (1966): Resection of the Thbal Shaft for Osteomyelitis in Children. Journal of Bone and Joint Surgery, 48-A, 1328.

Bryson, A. F., and Mandell, B. B. (1964): Primary Closure after Operative Treatment of Gross Chronic Osteomyelitis. Lancet, 1, 1179. 
Butler, E. C. B. (1940): The Treatment, Complications and Late Results of Acute Haematogenous Osteomyelitis. British Journal of Surgery, 28, 261.

CAPener, N., and Pierce, K. C. (1932): Pathological Fractures in Osteomyelitis. Journal of Bone and Joint Surgery, 14, 501.

Evans, A. J. (1914): Excision of the Diaphysis of the Humerus, with Full Functional Recovery. British Journal of Surgery, 1, 632.

Girdlestone, G. R., and Foley, W. B. (1933): Extensive Loss of Tibial Diaphysis. Tibio-fibular Grafting. British Journal of Surgery, 20, 467.

GrAY, G. M. (1928): Case of Osteomyelitis of Radius Treated by Bone-Grafting. British Journal of Surgery, 15,671 .

GreENwOOD, H. H. (1932): Reconstruction of Forearm after Loss of Radius. British Journal of Surgery, 20, 58.

Groves, E. W. H. (1921): On Modern Methods of Treating Fractures. Second edition, p. 320. Bristol: John Wright \& Sons Ltd.

HahN, E. (1884): Eine Methode, Pseudarthrosen der Tibia mit grossem Knochendefekt zur Heilung zu bringen. Centralblatt für Chirurgie, 11, 337.

Hazlett, J. W. (1954): The Use of Cancellous Bone Grafts in the Treatment of Subacute and Chronic Osteomyelitis. Journal of Bone and Joint Surgery, 36-B, 584.

Huntington, T. W. (1905): Case of Bone Transference. Annals of Surgery, 41, 249.

JONES, R. W. (1934): Reconstruction of Forearm after Loss of Radius. British Journal of Surgery, 22, 23.

Lowe, H. G. (1963): Radio-ulnar Fusion for Defects in the Forearm Bones. Journal of Bone and Joint Surgery, 45-B, 351.

LuCAS, J. M. (1960): Huntington's Technic in Repair of Tibia Following Osteomyelitis. Philippine Journal of Surgery and Surgical Specialties, 15, 272.

Mowlem, R. (1944): Cancellous Chip Bone-Grafts. Lancet, 2, 746.

Murray, R. A. (1955): The One-Bone Forearm. Journal of Bone and Joint Surgery, 37-A, 366.

Parisien, V. (1963): Fibular Transfer for Tibial Defect. Bulletin of the Hospital for Joint Diseases, $24,142$.

Phemister, D. B. (1915): Subperiosteal Resection in Osteomyelitis. Journal of the American Medical Association, 65, 1994.

Platt, H. (1928): Treatment of Acute Osteomyelitis. Proceedings of the Royal Society of Medicine, $21,1377$.

Pyrah, L. N., and Pain, A. B. (1933): Acute Infective Osteomyelitis. British Journal of Surgery, $20,590$.

Roper, A. (1964): In Priorities in Orthopaedic Surgery. Proceedings of First Symposium on Rehabilitation in Africa, p. 44. London: National Fund for Research into Poliomyelitis and Other Crippling Diseases.

Ruskowski, Y. (1965): Traitement chirurgical des pertes de substance du tibia par implantation du péroné. Revue de chirurgie orthopédique et réparatrice de l'appareil moteur, 51, 719.

Thomson, J., and Lewis, I. C. (1950): Osteomyelitis in the Newborn. Archives of Disease in Childhood, $25,273$.

TruetA, J. (1959): The Three Types of Acute Haematogenous Osteomyelitis. Journal of Bone and Joint Surgery, 41-B, 671.

Trueta, J., and Morgan, J. D. (1954): Late Results in the Treatment of One Hundred Cases of Acute Haematogenous Osteomyelitis. British Journal of Surgery, 41, 449.

Vitale, C. C. (1952): Reconstructive Surgery for Defects in the Shaft of the Ulna in Children. Journal of Bone and Joint Surgery, 34-A, 804.

Whrte, M. (1935): Some Bone and Joint Conditions in Children. Glasgow Medical Journal, 124, 1.

WiLson, P. D. (1941): A Simple Method of Two-stage Transplantation of the Fibula for Use in Cases of Complicated and Congenital Pseudarthrosis of the Tibia. Journal of Bone and Joint Surgery, 23, 639. 